\title{
Ship-in-a-Bottle Biomicrochips Fabricated by Hybrid Femtosecond Laser Processing
}

\author{
Koji Sugioka, Dong Wu, and Katsumi Midorikawa \\ RIKEN, Wako, Saitama 351-0198, Japan \\ ksugioka@ riken.jp
}

\begin{abstract}
We demonstrate fabrication of highly functional biomicrochips by hybrid femtosecond laser processing. In this process, 3D microfluidic structures are first formed inside photosensitive glass by femtosecond laser direct writing followed by thermal treatment and successive chemical wet etching. Then, functional microcomponents are integrated inside the fabricated microfluidic structures by two-photon photopolyerization. We term the fabricated microchips ship-in-a-bottle biomicrochips,
\end{abstract}

\section{Introduction}

Femtosecond laser processing is a promising technique for fabrication of biomicrochip since it can be used to modify the interior of glass in a spatially selective manner due to multiphoton absorption and thereby to directly form 3D microfluidic structures inside substrates [1-4]. We developed a technique fabricating true three-dimensional (3D) microfluidic structures inside photosensitive glass by femtosecond laser direct writing followed by thermal treatment and successive chemical wet etching. This technique can also integrate some kinds of microoptical components and fluid control microcomponents. Then, this technique was successfully applied to fabricate functional microfluidics and optofluidics for determining the functions of microorganisms [2, 4-6]. In the meanwhile, this technique is somewhat weak in integrating complex microcomponents with micro and nano feature sizes in the microfluidics due to its limited spatial resolution and top-down processing nature. This paper proposes a novel strategy (hybrid femtosecond laser processing) which combines two femtosecond laser processes, i.e., femtosecond laser modification followed by wet etching and two-photon photopolymerization (TPP) to overcome this problem and thereby realize highly functional biomicrochips.

\section{Experimental procedure}

Figure 1 shows the experimental procedure of hybrid femtosecond laser processing. A second harmonic (523 nm) from commercial femtosecond laser (wavelength: $1045 \mathrm{~nm}$; pulse width: $370 \mathrm{fs}$; repetition rate: $200 \mathrm{kHz}$ ) was used in our experiment. Firstly, we fabricated 3D embedded microfluidic structures inside photosensitive glass by femtosecond direct writing (Fig. 1(a)) followed by thermal treatment (Fig. 2(b)) and successive chemical wet etching using a hydrofluoric acid solution (Fig. 1(c)). Then, the fabricated microfluidic structures were filled up with the resin SU-8 (Fig. 1(d)). After pre-baking at $95^{\circ} \mathrm{C}$ for $1 \mathrm{~h}$, TPP was carried out (Fig. 1(e)). By developing the laser exposed regions, 3D micro and nanostructures were formed inside the microfluidics (Fig. 1(d)). We termed the glass microfluidics integrated with 3D polymer micro and nanostructures a ship-in-a-bottle biomicrochip.

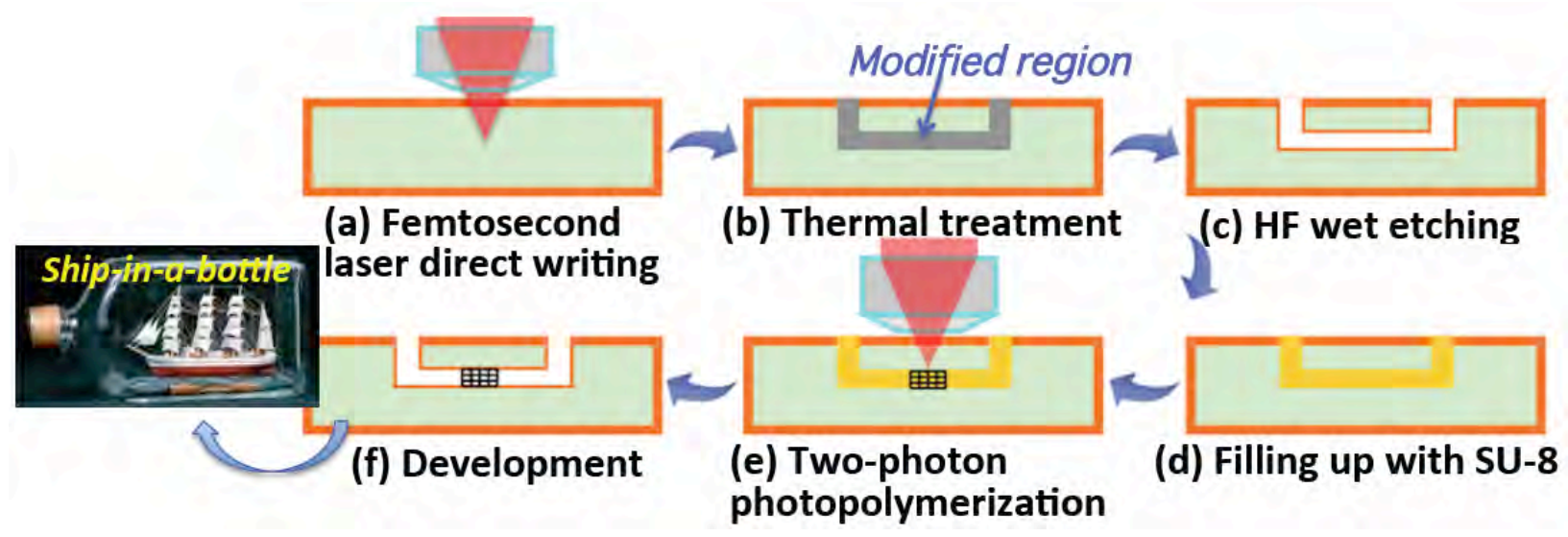

Fig. 1. Fabrication procedure of a ship-in-a-bottle biomicrochip by hybrid femtosecond laser processing. 


\section{Experimental results}

The hybrid femtosecond laser processing was used to fabricate microfluidics integrated with micro/nanofilters and micromixers. As one of examples, Fig. 2 shows integration of the micromixer in the Y-shaped microfluidic channel ((a) principle of a designed micromixer, (b) an SEM image of micromixer structure fabricated on the glass surface by TPP). Microfluidic channels whose diameters are in a range of hundreds nm to hundreds $\mu \mathrm{m}$ usually produce laminar flow of fluids since the Raynolds number for such a dimension is low [7], as shown in Fig. 2 (c). Efficient mixing of two or more kinds of fluids in the microfluidics is a crucial issue in biochip applications. In Fig. 2 (d), the same structure as shown in Fig. 2 (b) was formed at the downstream of junction inside the microfluidic channel (indicated by an arrow), performing efficient mixing of two different kinds of fluids. The mixing efficiency was evaluated to be as high as $87 \%$.
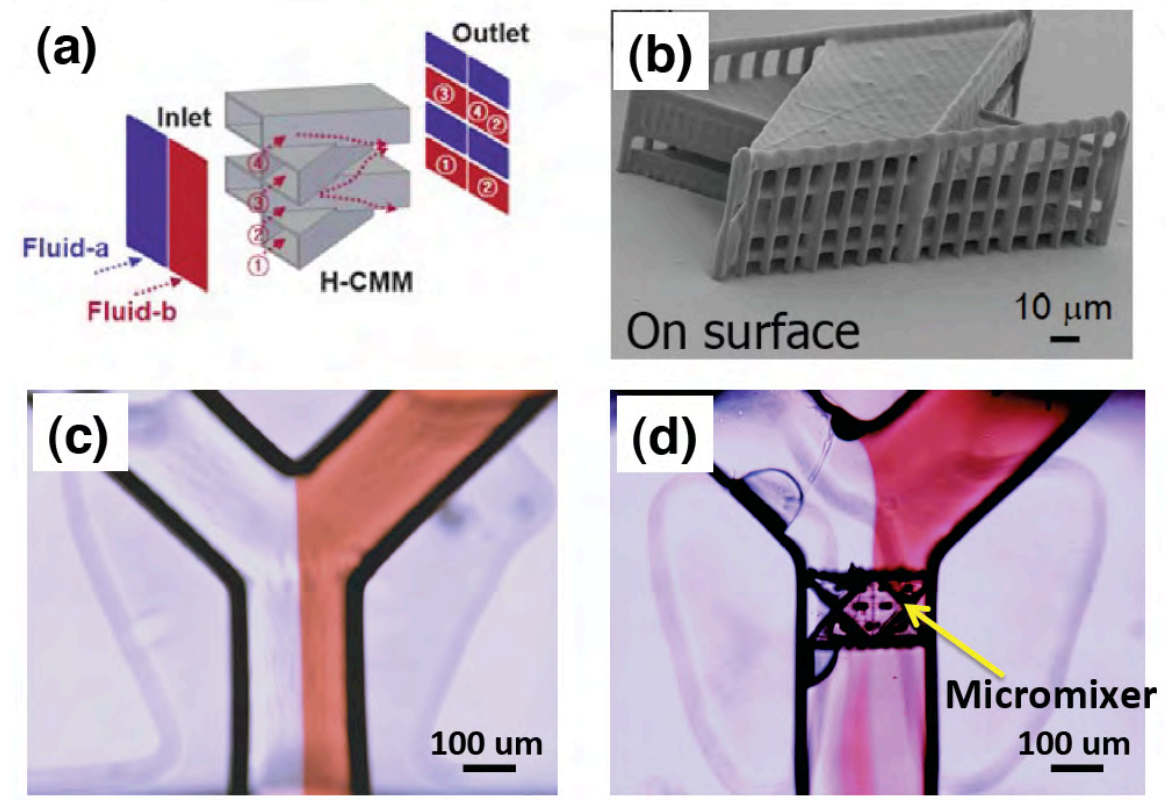

Fig. 2 (a) Principle of designed micromixer, (b) SEM image of micromixer structure fabricated on glass surface by TPP, (c) formation of laminar flow in the Y-shaped microfluidic channel, and (d) performance of fluid mixing in the microfluidic channel integrated with the micromixer .

\section{Summary}

We proposed a new technique referred to as hybrid femtosecond laser processing for fabrication of highly functional biomicrochips. The proposed technique was applied to fabricate microfluidics integrated with fluid control microcomponents. The fabricated microchip demonstrated high performance in fluid mixing. This technique is very promising for fabricating a variety of microfluidics and optofluidics with multi functions that cannot be realized by the existing technologies.

\section{References}

[1] A. Crespi, Y. Gu, B. Ngamsom, H. J. W. M. Hoekstra, C. Dongre, M. Pollnau, R. Ramponi, H. H. van den Vlekkert, P. Watts, G. Cerullo and R. Osellame, "Three-dimensional Mach-Zehnder interferometer in a microfluidic chip for spatially-resolved label-free detection," Lab Chip 10, $1167-1173(2010)$

[2] K. Sugioka and Y. Cheng, "Integrated microchips for biological analysis fabricated by femtosecond laser direct writing," MRS Bulletin 36, 1020-1027 (2011).

[3] A. Schaap, T. Rohrlack and Y. Bellouard, "Optical classification of algae species with a glass lab-on-a-chip," Lab Chip 12, 1527-1532 (2012).

[4] K. Sugioka and Y. Cheng, “ Femtosecond laser processing for optofluidic fabrication,” Lab Chip 12, 3576-3589 (2012).

[5] K. Sugioka, Y. Hanada, and K. Midorikawa, "Three-dimensional femtosecond laser microm-achining of photosensitive glass for biomicrochips," Laser \& Photon. Rev. 4, 386-400 (2010).

[6] Y. Hanada, K. Sugioka, I. S-Ishikawa, H. Kawano, A. Miyawaki, K. Midorikawa, "3D microfluidic chips with integrated func-tional microelements fabricated by a femtosecond laser for studying the gliding mechanism of cyanobacteria," Lab Chip 11, 2109-2115 (2011). [7] http://www.kirbyresearch.com/index.cfm/page/ri/ufluids.htm. 